\title{
ALGUNAS ESPECIES DEL GENERO CONOCYBE FAYOD QUE FRUCTIFICAN EN LOS JARDINES Y PARQUES DE LA PROVINCIA DE MADRID
}

\author{
G. MORENO (*)
}

\section{RESUMEN:}

Se estudian macro y microscópicamente tres especies del género Conocybe Fayod, recolectadas en ambientes artificiales (jardines) en Madrid, son: C. cylindracea Maire \& Kühner ix Sing., C. lactea (Lange) Métrod y C. rickenii (J. Schäeffer) Kühner, de los cuales la primera especie solo se conoce actualmente del Norte de Africa (Marruecos), y la última citada en Europa es nueva para el Catálogo micológico español.

\section{SUMMARY:}

Three species of the Conocybe Fayod genus were studied macro and microscopically. They were collected in artificial Madrid environments (gardens) and we found that they be long to the following species: C. cylindracea, C. lactea, C. rickenii. The first one has only been described on Norther Africa (Maroc), and the last one, has al ready been described in Europe, but it is a new specie for the Spanish mycological catalogue.

\section{INTRODUCCION}

El género Conocybe Fayod, Prodrome Ann. Sc. Nat. Bot. 7 (9): 357, (1889), agrupa a pequeñas especies encuadradas en la Familia Bolbitiaceae Sing. Pap. Mich. Acad.Sc. Arts. Lett. 32: 147 (1948), que presentan la cutícula formada por células piriformes a claviformes, entre las que es frecuente observar pileocistidios o células diferentes morfológicamente, alargadas, capitadas o no. Las láminas no poseen estrato medio y sin embargo tienen el subhimenio bien desarrollado, presentan cistidios faciales y marginales generales capitados.

Ecológicamente se presentan algunas especies de este género como coprófilas, Moreno (1976), Moreno y Barrasa (1977) o bien viviendo sobre restos de plantas herbáceas, comportándose la gran mayoría como pratícolas, Kühner (1935), Singer (1975), algunas son muy raramente lignícolas Kühner-Romagnesi (1953). Las reco-

* Cátedra de Botánica. Facultad de Farmacia. Universidad de Alcalá de Henares (Madrid). 
lectas pratícolas es decir en zonas de pastizal, fructifican sobre las hojas y raíces muertas de diferentes Poaceas, lo que hace se incluyan en el gran grupo de las humícolas, representando a especies que se desarrollan sobre material orgánico en descomposición y no siendo raro recolectarlas en humus de diferentes procedencias, como restos de hojas de árboles caducifolios, hojas de plantas perennantes...

Es frecuente observar en primavera, verano y otoño la fructificación de diversas especies en los jardines y lugares recientemente estercolados, con vegetación de Poaceas, a estos ámbitos artificiales hemos prestado atención y hemos recolectado diferentes especies, describimos algunas de ellas en la presente nota, y mantenemos actualmente en estudio diferentes recolectas del grupo C. tenera Schff. ex Fr., aún no muy bien delimitadas taxonómicamente.

Conocybe cylindracea R. Maire-Kühner ex Singer.

三 C. cylindracea R. Maire-Kühner, Le genre Galera p. 114-115 (1935).

Sombrero elíptico a cilíndrico, conservando esta posición y no abriéndose, mide $0,6-1,2 \times 0,3-0,7 \mathrm{~cm}$. de color marrón a marrón grisáceo con el ápice más oscuro, el margen no es estriado, no es higrófano. Pie de 4-6 $\times 0,1-0,15 \mathrm{~cm}$., cilíndrico, pruinoso, algo mas ensanchado en la base, donde suele curvarse, de color marrón oscuro. Láminas amarillo ocre, ascendentes y adnadas.

Esporas de 9-12 × 6-8, 5 u. anchamente elípticas, operculadas, con apícula muy saliente. Basidios tetraspóricos, claviformes. Cistidios en forma de cabeza de alfiler, parte inferior ventruda, miden $16-20 \times 8-9 \times 3,5-5 \mathrm{u}$. Revestimiento del pie formado por filamentos alargados y no capitados, mas o menos abundantes por todo él y a veces fasciculados.

Habitat: Una recolecta de media docena de carpóforos, en los jardines del Museo de Arte Contemporáneo en la Ciudad Universitaria de Madrid. Leg. C. Ladó, J. A. Barberá y G. Moreno (10-IX-75) n. ${ }^{\circ}$ herbario propio 321.

Distribución: Solamente aparece citado en la bibliografía que hemos podido consultar en Africa del Norte (Marruecos), Malençon y Bertault (1970), país de donde fue descrito por Maire y Kühner (1935). En comunicación personal Bon (1977), nos indica igualmente que solo conoce la corología dada por Malençon y Bertault para esta especie en 1970.

Observaciones: Especie fácil de identificar por su pie cubierto de pelos no capitados y su sombrero fuertemente cilindráceo.

Conocybe lactea (Lange) Métrod.

$=$ C. lateritia (Fr.) ss. Kühn. Le genre Galera p. 121-124 (1935).

$=$ C. lateritia Fr. Syst. Myc. p. 265 (1821).

Sombrero de $2-5 \mathrm{~cm}$. de diámetro, elíptico a campanulado abriéndose en la madurez para tomar una forma anchamente acampanada, margen recurvado no 
siendo estriado, opaco, no higrófano, Varía del color blanquecino a débilmente ocráceo, en la vejez se readsorbe el carpóforo con facilidad. Pie cilíndrico de 5-12 $\times 0,1-0,3 \mathrm{cms}$, ensanchado en la base en un pequeño bulbo de color blanco como todo él, llegando a tener hasta $0,8 \mathrm{~cm}$. de diámetro, presenta unas estriaciones longitudinales típicas y está recubierto por una pruina característica formada por pelos a la lupa. Láminas muy estrechas, blancas en un principio, pasando al color ocre en la madurez, adnadas y ascendentes.

Esporas elípticas, operculadas, ensanchadas a veces en la parte basal y por lo tanto ovales, miden de 12-14 × 7-9 u. Basidios claviformes tetraspóricos. Cistidios marginales en forma de cabeza de alfiler.

Revestimiento del pie formado por filamentos filiformes hialinos, cubriendo toda la superficie y formando frecuentemente densos fascículos.

Habitat: Éntre la hierba en diversos jardines de Madrid, recolectados en los meses de junio y julio principalmente, poseemos muestras de herbario de los jardines de la Facultad de Farmacia de Madrid, Facultad de Farmacia de Alcalá de Henares (Madrid), El Retiro Madrid, jardines de la calle Rosales (Madrid) y de jardines de Villaviciosa de Odón (Madrid). Leg. G. Moreno excepto en la última recolecta leg. F. D. Calonge, núms. de herbario propio 322, 1.191, 1.197, 950 y 324 respec.

Distribución: Aparece muy poco citado en los Catálogos micológicos españoles, se conoce de Cataluña, Maire (1933) (1937) y de la zona centro Calonge (1971).

Observaciones: Hemos recolectado abundantemente junto con el tipo, ejemplares con láminas blancas que forman esporas sin pigmentación ocrácea, poseyendo cistidios faciales y marginales igual al tipo, con el pie típicamente blanco y recubierto de pelos sin capitar, debiéndose posiblemente a mutaciones, como pudimos comprobar al ponernos en comunicación con los Profs. H. Romagnesi (París) y M. Bon (Lille), formas que Kühner (1935) denomina estériles y abundantes junto con la normal. Hemos estudiado de esta forma las recolectas siguientes: jardines Fac. Farmacia (Madrid) Leg. M. T. Telleria y G. Moreno (15-VI-75) n. ${ }^{\circ}$ herbario 325, jardín Ciudad Universitaria (Madrid) Leg. G. Moreno (10-III-74) n. ${ }^{\circ} 326$, jardines Villaviciosa de Odón (Madrid) Leg. F. D. Calonge (10-VII-74) n. 327.

Conocybe rickenii (J. Schff.) Kühn. Le genre Galera p. 115-120 (1935). = Galera pygmaeoaffinis Ricken, Blatterpilze p. 225 (1915).

$=G$. siliginea Bres., Iconographia; pl. 807 (1931).

Sombrero variando de cónico a hemisférico e incluso acampanado, no quedando plano en general, de 1-3 cm. de diámetro, de color ocráceo, estriado por transparencia, fuertemente higrófano. Pie cilíndrico algo más ensanchado en la base, mide de 2-6 x 0,1-0,15 cm., recubierto todo él por una pruinosidad característica blanquecina, variando a color ocráceo al rozarlo con el dedo o en la vejez, el inte- 
rior del pie (carne) está formado por hifas de color ocráceo a miel pálido. I.aminillas ascendentes, apretadas de color ocráceo.

Esporas elípticas, operculadas a veces, miden de 14-17 $\times 8,5-10 \mathrm{u}$., de pared espesa, lisas y de color amarillo dorado al microscopio, tomando color rojizo en amoniaco. Basidios bispóricos. Cistidios marginales abundantes en forma de cabeza de alfiler con la base ventruda, miden de 18-30 ×7-11 ×4-6u. Revestimiento del pie formado por pelos hialinos cubriendo toda su superficie, variando en longitud y grosor.

Habitat: Entre la hierba de diversos jardines de Madrid, conservamos material en herbario del jardín del Museo de Arte Contemporáneo de la Ciudad Universitaria (Madrid) Leg. J. A. Barberá, C. Ladó y G. Moreno (10-IX-75) n. ${ }^{\circ}$ herbario propio 330 y 331. En la tierra estercolada de una maceta Leg. K. Tabba (3-IV-76) n. ${ }^{\circ}$ 329. sultada.

Distribución: No aparece citado en la bibliografía española por nosotros con-

Observaciones: El hecho de presentar cistidios marginales en forma de cabeza de alfiler, hace se encuadre dentro de la sección Capitatae Kühner (1935), el tamaño sumamente grande de sus esporas, los basidios bispóricos y la presencia de pelos hialinos no capitados en el pie, lo definen taxonómicamente.

\section{BIBLIOGRAFIA}

BARRASA, J. M. ${ }^{2}$ \& MORENO, G. 1978. Contribución al estudio de hongos que viven sobre materias fecales (2. a aportación). Acta Bot. Malacitana. (En prensa).

CALONGE, F. D. 1971. Estudio sobre hongos III. Anal. Inst. Bot. A. J. Cavanilles 28: 7-33.

KÜHNER, R. 1935. Le genre Galera Fr. Ed. P. Lechevalier. Paris 240 p.

KÜHNER, R. \& ROMAGNESI, H. 1953. Flore analytique des champignons superiers, Masson et Cie. Paris $556 \mathrm{p}$.

MAIRE, R. CODINA, J. \& FONT-QUER, P. 1933. Fungi Catalaunici. Contributions à l'étude de la Flore mycologique de la Catalogne. Pub. Junta Cienc. Nat. de Barcelona., 15 (2). Barcelona.

MAIRE, K. 1937. Fungi Calalaunici. Contributions à l'étude de la Flore mycologique de la Catalogne. Pub. Inst. Bot. 3 (4). Barcelona.

MALENÇON, G. \& BERTAULT, R. 1970. Flore des champignons superieurs de Maroc. I. Rabat.

MORENO, G. 1976. Contribución al estudio micológico de Andalucía. Nota I, Agaricales nuevos o raros para España peninsular. Acta Bot. Malacitana 2: 5-20. Málaga.

MORENO, G. \& BARRASA, J. M. ${ }^{2}$ 1977. Contribución al estudio de hongos que viven sobre materias fecales (1. a aportación). Acta Bot. Malacitana 3: 5-33. Málaga.

MOSER, M. 1967. Basidiomyceten II. Teil Dei Röhrlinge und Blätterpilze (Agaricales) Gustav Fischer Verlag. Stuttgart.

SINGER, R. 1975. The Agaricales in modern taxonomy. Straub \& Cramer. Germany 912 p. 


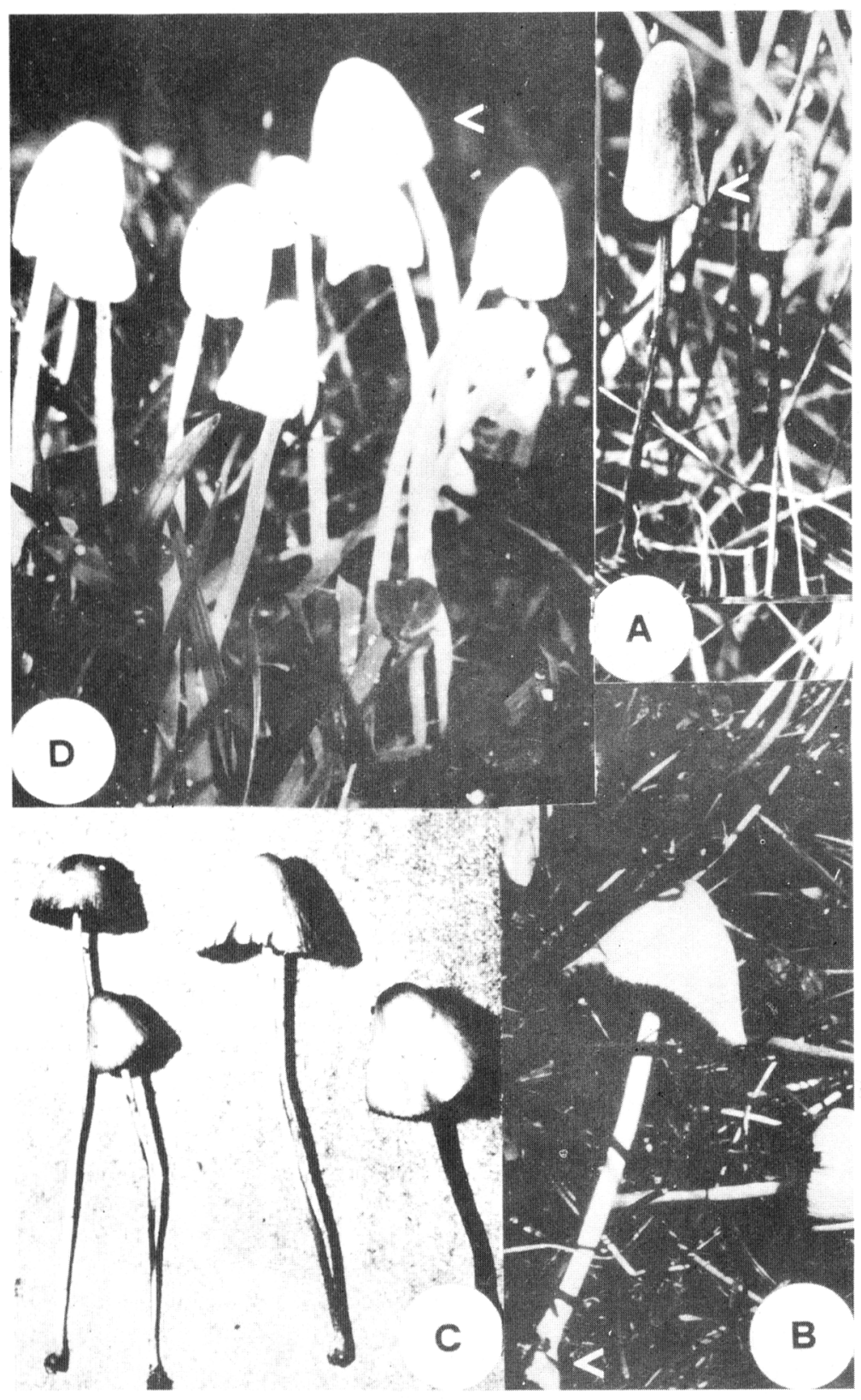

Lám. 1.-A. Conocybe cylindracea Maire \& Kühner ex Singer, detalle carpóforos; B. C. lactea (Lange) Métrod, detalle carpóforo y base del pie; C. C. rickenii (J. Schff.) Kühn., detalle carpóforos; D. Formas estériles de C. lactea. 


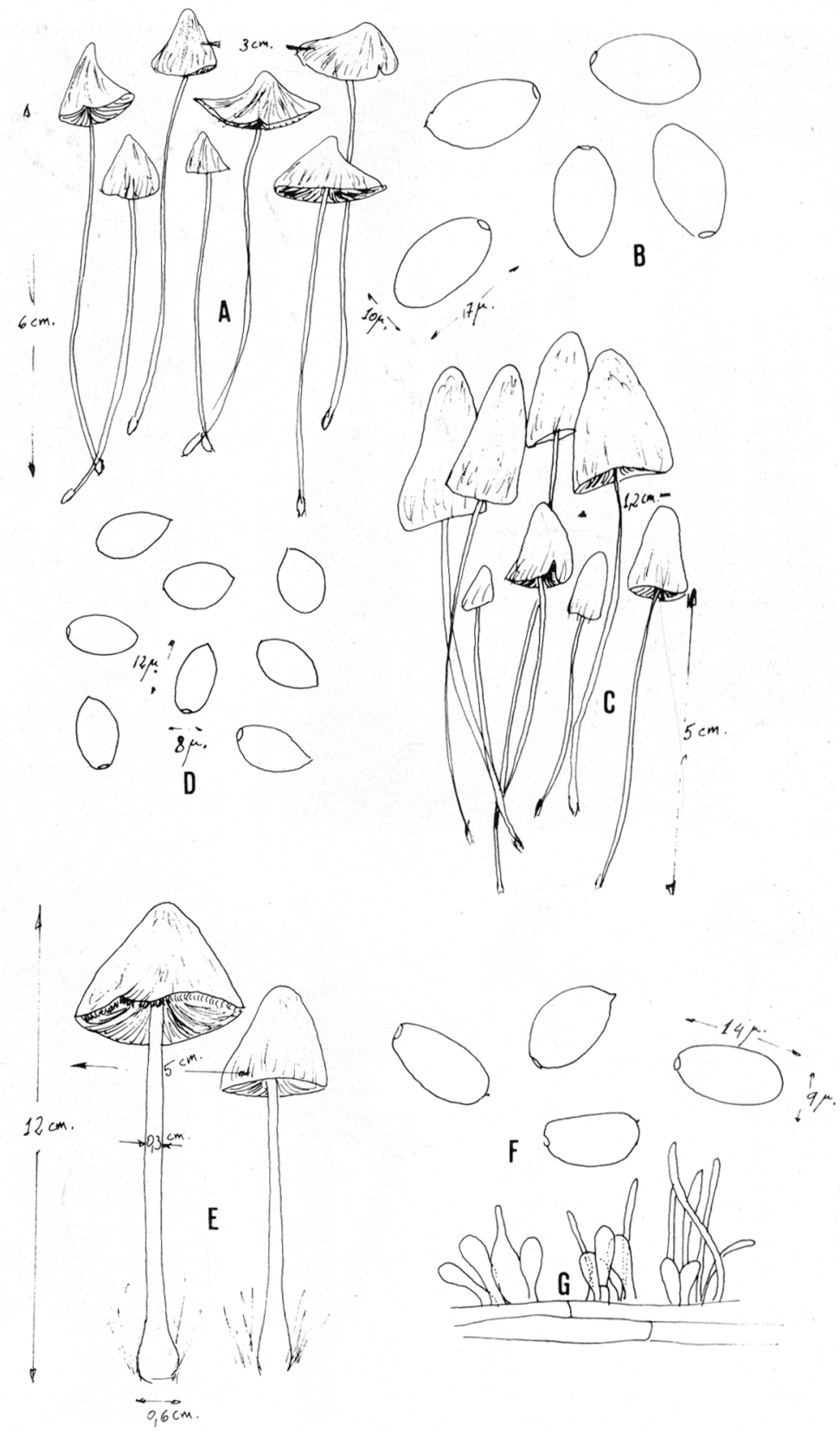

Lám. 2.-Conocybe rickenii (J. Schff.) Kühn., A. Carpóforos, B. Esporas; C. cylindracea Maire \& Kühner ex Singer, C. Carpóforos, D. Esporas; C. lactea (Lange) Métrod, E. Carpóforos, F. Esporas, G. Detalles pelos del pie. 